\title{
Electronic States in a Doubly Eccentric Cylindrical Quantum Wire
}

\author{
R. Kumar ${ }^{1 *}$, S. N. Singh ${ }^{2}$ \\ ${ }^{1}$ Department of Physics, St. Xavier's College, Ranchi-834001, India \\ ${ }^{2}$ Department of Physics, Ranchi University, Ranchi-834001, India
}

Received 15 February 2020, accepted in final revised form 5 June 2020

\begin{abstract}
Electronic states of a single electron in doubly eccentric cylindrical quantum wire are theoretically investigated in this paper. The motion of electron in quantum wire is free along axial direction in a cylindrical quantum wire and restricted in annular regions by three different parallel finite cylindrical barriers as soft wall confinement. The effective mass Schrödinger equation with effective mass boundary conditions is used to find energy eigenvalues and corresponding wavefunctions. Addition theorem for cylindrical Bessel functions is used to shift the origin for applying boundary conditions at different circular boundaries. Fourier expansion is applied after addition theorem to get wavefunctions in analytical form. A determinant equation is obtained as a result of applications of effective mass boundary conditions which roots gives energy of various electronic states. The lowest root gives ground state energy. The variation in ground state energy with eccentricity is obtained numerically and presented graphically. Electronic states in massive wall confinement and hard wall confinement is further obtained as limiting behavior of the states obtained in soft wall confinement. The knowledge of electronic states in such cylindrical hetrostructures semiconductor material can lead to improve the efficiency of many quantum devices.
\end{abstract}

Keywords: Doubly eccentric quantum wire; Addition theorem; Effective mass Schrödinger equation; Soft wall confinement; Bessel functions.

(C) 2020 JSR Publications. ISSN: 2070-0237 (Print); 2070-0245 (Online). All rights reserved. doi: http://dx.doi.org/10.3329/jsr.v12i4.45504 J. Sci. Res. 12 (4), 473-483 (2020)

\section{Introduction}

The semiconductor hetrostructures are very useful in construction of many quantum devices like quantum laser where active medium is a quantum wire or dot. A lot of properties of quantum wire has been studied. Optical properties of a cylindrical quantum wire has been investigated theoretically to study variation of absorption coefficient and refractive index [1,2]. Binding energies of impurity in quantum dot structures have been studied as function of geometry [3]. Effect of shape and size on electron energy spectrum

${ }^{*}$ Corresponding author: rkumariitm@gmail.com 
in various shaped quantum structures have been investigated [4-8]. The properties of quantum wire and dots with cylindrical geometry are of special interests among various researchers due to its wide range of applications [9-15]. Theoretical study of electrical properties of single eccentric cylindrical structures with $\mathrm{C}_{1 \mathrm{v}}$ and $\mathrm{C}_{\infty \mathrm{v}}$ symmetries have been studied [16]. We have investigated electrical properties of a new structure-doubly eccentric cylindrical quantum wire. There is no report on electronic states of a doubly eccentric cylinder in nano regime to our knowledge. A doubly eccentric cylindrical quantum wire consists of two eccentric cylindrical quantum wire nested inside a cylindrical quantum wire of larger radius. A cross-section of such quantum wire is shown in Fig.1. Such doubly eccentric cylindrical wire in nano regime can help to develop semiconductor material to enhance the efficiency of many quantum devices. The electronic states in a doubly cylindrical hetrostructures with $\mathrm{C}_{2 \mathrm{v}}$ symmetry in soft wall confinement (SWC) are studied theoretically in this paper. As its limiting behavior, the corresponding electronic states in hard wall confinement (HWC) and massive wall confinement (MWC) are also obtained. An exact solution of effective mass Schrödinger equation is obtained by using addition theorem for shifting origin of cylindrical Bessel functions in hetrostructures to satisfy corresponding boundary conditions for wavefunctions. The validity of addition theorem in doubly eccentric cylinder imposes a restriction on the range of its eccentricity but that doesn't lead to an issue as allowed range of eccentricity to cover most of practical problems.

Section 2 of this paper presents general geometrical structure of the problem and its exact solution in soft wall confinement (SWC). At first, the solution of effective mass Schrödinger equation is obtained in terms of cylindrical Bessel functions for various regions. Effective mass boundary conditions are then applied across each pair of boundaries. Fourier expansion is applied on the expression obtained after applying appropriate boundary condition to get the solution in analytical form. As a result, these give a set of infinite simultaneous equations which is discussed in section 3. A determinant equation is obtained for non-trivial solution of the infinite simultaneous equations. The roots of this determinant equation give various energy eigenvalues. The lowest root of determinant equation is obtained numerically to get ground state energy. The separation between axes of two eccentric cylinders is termed as eccentricity of structure. The ground state energy is calculated by varying eccentricity of structure. The result in variation of ground state energy with eccentricity is presented graphically. The corresponding limiting solution for massive wall confinement (MWC) and hard wall confinement (HWC) is discussed in section 4. Section 5 presents concluding remarks.

\section{Solution}

Fig. 1 shows annular cross-section of a doubly eccentric cylindrical structure. The length is along Z-axis which is perpendicular to plane of figure. The axes of region I, II and III are parallel. Radii of regions I and III are ' $a$ ' and that of region II is ' $b$ '. The separation between centres of region II and III is $d_{1}$ and that between I and II is $d_{2}$. In the structure, it 
is kept $d_{1}=d_{2}=d$ so that structure has $C_{2 \mathrm{v}}$ symmetry which is more useful in physical applications. However, this methodology can be used to study the electronic states in doubly eccentric cylinder with $d_{1} \neq d_{2}$ as well. The parameter ' $d$ ' represents separation between axes of inner cylinder and is called eccentricity.

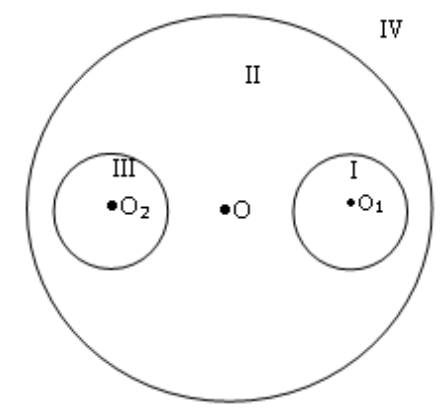

Fig. 1. Cross-section of a doubly eccentric cylinder.

We have considered a system in which an electron is free to move along axis of cylinder in region II and its annular motion is confined by different cylindrical barriers shown as regions I, III and IV in Fig. 1. Using the theory of effective mass approximation, each region can be considered as region of constant potential with corresponding effective mass of the electron. Let effective mass of the electron in $l^{\text {th }}$ region is $m_{l}^{*}, l=1,2,3$ and 4. We have set the value of potential energy of region II equal to zero as the reference of potential energy. Let the barrier potential in $l^{\text {th }}$ region is $U_{l}(>0), l=1,3$ and 4.Taking $\left(\rho_{1}, \varphi_{1}, z_{1}\right)$ as cylindrical polar co-ordinates with respect to origin $\mathrm{O}_{1}$, the effective mass Schrödinger equation [17-18] for hetrostructures is given as

$$
\left[-\frac{\hbar^{2}}{2} \nabla\left\{\frac{1}{m^{*}\left(\rho_{1}, \varphi_{1}, z_{1}\right)} \nabla\right\}+U\left(\rho_{1}, \varphi_{1}, z_{1}\right)\right] \psi\left(\rho_{1}, \varphi_{1}, z_{1}\right)=E \psi\left(\rho_{1}, \varphi_{1}, z_{1}\right)
$$

For uniform distribution of effective mass $m_{l}{ }_{l}$ in the $l^{\text {th }}$ region, $m_{l}{ }_{l}\left(\rho_{1}, \varphi_{1}, z_{1}\right)=m_{l}{ }_{l}$, the Eq. (1) takes the form

$$
\nabla^{2} \psi\left(\rho_{1}, \varphi_{1}, z_{1}\right)=-\frac{2 m^{*}(E-U)}{\hbar^{2}} \psi\left(\rho_{1}, \varphi_{1}, z_{1}\right)
$$

In region I, the solution of Eq. (2) with origin at $\mathrm{O}_{1}$ comes as

$$
\begin{aligned}
& \psi_{I}\left(\rho_{1}, \varphi_{1}, z_{1}\right)=\sum_{m=0}^{\infty} A_{m} I_{m}\left(k_{1} \rho_{1}\right) \sin \left(m \phi_{1}+\phi_{0}\right) e^{i \beta z_{1}} \\
& \text { with } k_{1}^{2}=-\frac{2 m_{1}^{*}\left(E-U_{1}\right)}{\hbar^{2}}+\beta^{2}
\end{aligned}
$$

where $A_{m}$ are unknown constants, $I_{m}\left(k_{1} \rho_{1}\right)$ is modified Bessel function of first kind, $\beta$ is axial wave number and $\phi_{0}=0$ gives odd parity and $\phi_{0}=\frac{\pi}{2}$ gives even parity.

In region II, the solution of Eq. (2) with origin at $\mathrm{O}_{1}$ comes as 


$$
\begin{aligned}
& \psi_{I I}\left(\rho_{1}, \varphi_{1}, z_{1}\right)=\sum_{m=0}^{\infty}\left(B_{m} J_{m}\left(k_{2} \rho_{1}\right)+C_{m} Y_{m}\left(k_{2} \rho_{1}\right)\right) \sin \left(m \phi_{1}+\phi_{0}\right) e^{i \beta z_{1}} \\
& \text { with } k_{2}^{2}=\frac{2 m_{2}^{*} E}{\hbar^{2}}-\beta^{2}
\end{aligned}
$$

where $B_{m}$ and $C_{m}$ are unknown constants, $J_{m}\left(k_{2} \rho_{1}\right)$ is Bessel function of first kind and $Y_{m}\left(k_{2} \rho_{1}\right)$ is Bessel function of second kind.

In region III, the solution of Eq. (2) with origin at $\mathrm{O}_{1}$ comes as

$\psi_{\text {III }}\left(\rho_{1}, \varphi_{1}, z_{1}\right)=\sum_{m=0}^{\infty}\left(D_{m} I_{m}\left(k_{3} \rho_{1}\right)+E_{m} K_{m}\left(k_{3} \rho_{1}\right)\right) \sin \left(m \phi_{1}+\phi_{0}\right) e^{i \beta z_{1}}$

with $k_{3}^{2}=-\frac{2 m_{3}^{*}\left(E-U_{3}\right)}{\hbar^{2}}+\beta^{2}$

where $D_{m}$ and $K_{m}$ are unknown constants, $I_{m}\left(k_{3} \rho_{1}\right)$ and $K_{m}\left(k_{3} \rho_{1}\right)$ are modified Bessel function of first and second kind respectively.

In region IV, the solution of Eq. (2) with origin at $\mathrm{O}_{1}$ comes as

$$
\begin{aligned}
& \psi_{I V}\left(\rho_{1}, \varphi_{1}, z_{1}\right)=\sum_{m=0}^{\infty} F_{m} K_{m}\left(k_{4} \rho_{1}\right) \sin \left(m \phi_{1}+\phi_{0}\right) e^{i \beta z_{1}} \\
& \text { with } k_{4}^{2}=-\frac{2 m_{4}^{*}\left(E-U_{4}\right)}{\hbar^{2}}+\beta^{2}
\end{aligned}
$$

Eq. (3) to Eq. (9) give the wavefunctions with some unknown constants in region I to IV respectively. These unknown constants and hence energy eigenvalues can be calculated using the fact that $\psi$ and $\frac{1}{m^{*}} \nabla \psi$ (effective mass boundary condition) has to be continuous at each of the boundaries [19]. To apply boundary conditions across boundary between region I and II, wavefunctions $\psi_{I}$ and $\psi_{I I}$ in terms of $\left(\rho_{1}, \varphi_{1}, z_{1}\right)$ with origin at $\mathrm{O}_{1}$ is good enough but for boundary between region II and III, wavefunctions $\psi_{\text {II }}$ and $\psi_{\text {III }}$ should be re-written in terms of new cylindrical polar co-ordinates $\left(\rho_{2}, \varphi_{2}, z_{2}\right)$ with respect to origin at $\mathrm{O}_{2}$. Again for boundary between region II and IV, wavefunctions $\psi_{I I}$ and $\psi_{I V}$ should be re-written in terms of $(\rho, \varphi, z)$ with respect to origin at $\mathrm{O}$. Addition theorem has been used to write the mathematical expression of wavefunction with origin shifted at ' $\mathrm{O}$ ' or ' $\mathrm{O}_{2}$ from ' $\mathrm{O}_{1}$ ' as required during application of boundary conditions [20-23].

\subsection{Boundary conditions between region I and II}

The two boundary conditions required to be satisfied at $\rho_{1}=a_{\text {for all }} \varphi_{1}$ and $z_{1}$ are

$$
\psi_{I}=\psi_{I I}
$$




$$
\text { and } \frac{1}{m_{1}{ }^{*}} \nabla \psi_{I}=\frac{1}{m_{2}{ }^{*}} \nabla \psi_{I I}
$$

Substituting Eq. (3) and Eq. (5) into Eq. (11) gives for each $\mathrm{m}$

$$
A_{m}=\frac{B_{m} J_{m}\left(k_{2} a\right)+C_{m} Y_{m}\left(k_{2} a\right)}{I_{m}\left(k_{1} a\right)}
$$

and substituting Eq. (3) and Eq.(5) into Eq. (12) gives for each $\mathrm{m}$

$$
B_{m}=C_{m} \frac{H_{m}\left(k_{1}, k_{2}\right)}{G_{m}\left(k_{1}, k_{2}\right)}
$$

where

$$
G_{m}\left(k_{1}, k_{2}\right)=\frac{1}{m_{1}^{*}}\left[k_{1} m_{2}^{*} I_{m}^{\prime}\left(k_{1} a\right) J_{m}\left(k_{2} a\right)-k_{2} m_{1}^{*} I_{m}\left(k_{1} a\right) J_{m}^{\prime}\left(k_{2} a\right)\right]
$$

and

$$
H_{m}\left(k_{1}, k_{2}\right)=\frac{1}{m_{1}^{*}}\left[k_{2} m_{1}^{*} I_{m}\left(k_{1} a\right) Y_{m}^{\prime}\left(k_{2} a\right)-k_{1} m_{2}^{*} I_{m}^{\prime}\left(k_{1} a\right) Y_{m}\left(k_{2} a\right)\right]
$$

\subsection{Boundary conditions between region II and III}

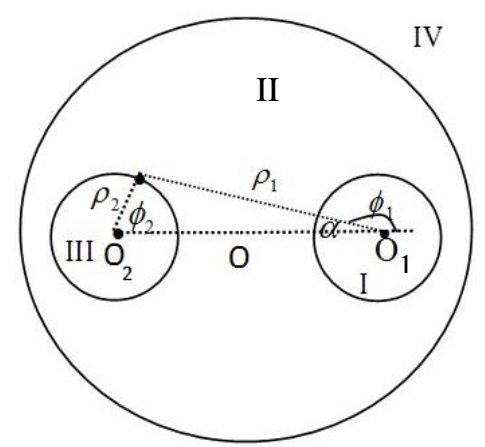

Fig. 2. For shift of origin from $\mathrm{O}_{1}$ to $\mathrm{O}_{2}$.

If $\left(\rho_{2}, \varphi_{2}, z_{2}\right)$ is cylindrical polar co-ordinate with origin at $\mathrm{O}_{2}$, the boundary conditions required to be satisfied at $\rho_{2}=a$ for all $\varphi_{2}$ and $z_{2}$ are

$$
\begin{aligned}
& \psi_{I I}=\psi_{I I I} \\
& \text { and } \frac{1}{m_{2}{ }^{*}} \nabla \psi_{I I}=\frac{1}{m_{3}^{*}} \nabla \psi_{I I I}
\end{aligned}
$$

To apply boundary conditions given by Eq. (17) and Eq. (18), we need to shift origin from $\mathrm{O}_{1}$ to $\mathrm{O}_{2}$ using addition theorem. Within the range of $d$ as $\frac{a}{2} \leq d \leq b-a$, the use of addition theorem with $\alpha=\pi-\phi_{1}$ (in Fig.2) gives 
$Z_{m}\left(k_{2} \rho_{1}\right) \sin \left(m \varphi_{1}+\varphi_{0}\right)=(-1)^{m+1} \cos \left(2 \phi_{0}\right) \sum_{p=-\infty}^{p=\infty} Z_{m+p}\left(k_{2} d_{12}\right) J_{p}\left(k_{2} \rho_{2}\right) \sin \left(p \varphi_{2}+\varphi_{0}\right)$,

where $Z=J$ or $Y$.

$K_{m}\left(k_{3} \rho_{1}\right) \sin \left(m \varphi_{1}+\varphi_{0}\right)=(-1)^{m+1} \cos \left(2 \phi_{0}\right) \sum_{p=-\infty}^{p=\infty} K_{m+p}\left(k_{3} d_{12}\right) I_{p}\left(k_{3} \rho_{2}\right) \sin \left(p \varphi_{2}+\varphi_{0}\right)$

and $I_{m}\left(k_{3} \rho_{1}\right) \sin \left(m \varphi_{1}+\varphi_{0}\right)=(-1)^{m+1} \cos \left(2 \phi_{0}\right) \sum_{p=-\infty}^{p=\infty}(-)^{p} I_{m+p}\left(k_{3} d_{12}\right) I_{p}\left(k_{3} \rho_{2}\right) \sin \left(p \varphi_{2}+\varphi_{0}\right)$

Using Eq. (5) and Eq. (19), the wavefunctions in region II about origin $\mathrm{O}_{2}$ comes as

$\psi_{I I}\left(\rho_{2}, \varphi_{2}, z_{2}\right)=$

$\sum_{m=0}^{\infty} \sum_{p=-\infty}^{p=\infty}(-1)^{m+1} \cos \left(2 \phi_{0}\right)\left[B_{m} J_{p+m}\left(k_{2} d_{12}\right)+C_{m} Y_{p+m}\left(k_{2} d_{12}\right)\right] J_{p}\left(k_{2} \rho_{2}\right) \sin \left(p \phi_{2}+\phi_{0}\right) e^{i \beta z_{2}}$

and using Eq. (20), Eq. (21) and Eq. (7), the wavefunction in region III about origin $\mathrm{O}_{2}$ comes as

$\psi_{\text {III }}\left(\rho_{2}, \varphi_{2}, z_{2}\right)=$

$\sum_{m=0}^{\infty} \sum_{p=-\infty}^{p=\infty}(-1)^{m+1} \cos \left(2 \phi_{0}\right)\left[(-)^{p} D_{m} I_{p}\left(k_{3} d_{12}\right)+E_{m} K_{p}\left(k_{3} d_{12}\right)\right] I_{p-m}\left(k_{3} \rho_{2}\right) \sin \left(p \phi_{2}+\phi_{0}\right) e^{i \beta z_{2}}$

Substituting the $\psi_{I I}$ and $\psi_{I I I}$ given by Eq. (22) and Eq. (23) into boundary conditions given by Eq. (17) and Eq. (18) and then expanding $\sin \left(p \phi_{2}+\phi_{0}\right)$ as Fourier series in $\phi_{2}$ and then using Eq.(14) yields a set infinite simultaneous equations in $C_{\mathrm{m}}$ 's as

$$
\sum_{n=0}^{\infty} \sum_{m=0}^{\infty} P_{m n} C_{m}=0
$$

where

$$
\begin{aligned}
& P_{m n}=\left[H_{m}\left(k_{1}, k_{2}\right) \alpha_{m n}\left(k_{2}, d_{12}\right)+G_{m}\left(k_{1}, k_{2}\right) \beta_{m n}\left(k_{2} d_{12}\right)\right] U_{n}\left(k_{2}, k_{3}\right) \\
& \text { with } U_{n}\left(k_{2}, k_{3}\right)=\left[k_{2} m_{3}{ }^{*} J_{n}^{\prime}\left(k_{2} a\right) I_{n}\left(k_{3} a\right)-k_{3} m_{2}{ }^{*} J_{n}\left(k_{2} a\right) I_{n}^{\prime}\left(k_{3} a\right)\right] \\
& \alpha_{m n}\left(k_{2} d_{12}\right)=\left[J_{n+m}\left(k_{2} d_{12}\right)+(-1)^{m-1} \cos \left(2 \phi_{0}\right) J_{n-m}\left(k_{2} d_{12}\right)\right] \\
& \text { and } \beta_{m n}\left(k_{2} d_{12}\right)=\left[Y_{n+m}\left(k_{2} d_{12}\right)+(-1)^{m-1} \cos \left(2 \phi_{0}\right) Y_{n-m}\left(k_{2} d_{12}\right)\right]
\end{aligned}
$$

\subsection{Boundary conditions between region II and IV}

If $(\rho, \varphi, z)$ is cylindrical polar co-ordinate with origin at $\mathrm{O}$, the boundary conditions required to be satisfied at $\rho=b$ for all $\phi$ and z are

$$
\psi_{I I}=\psi_{I V}
$$




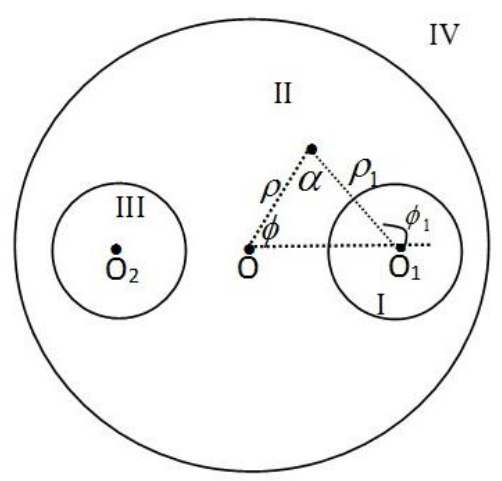

Fig. 3. For shift of origin from $\mathrm{O}_{1}$ to $\mathrm{O}_{2}$.

$$
\text { and } \frac{1}{m_{2}{ }^{*}} \nabla \psi_{I I}=\frac{1}{m_{4}^{*}} \nabla \psi_{I V}
$$

To apply boundary conditions given by Eq. (29) and Eq. (30) between region II and IV, $\psi_{I I}$ and $\psi_{I V}$ need to be written in cylindrical polar co-ordinates $(\rho, \varphi, z)$ with respect to origin at $\mathrm{O}$. Using addition theorem, the relation between Bessel functions in co-ordinates $(\rho, \varphi, z)$ and $\left(\rho_{1}, \varphi_{1}, z_{1}\right)$ with $\alpha=\phi_{1}-\phi$ (in Fig.3) comes as

$$
Z_{m}\left(k_{2} \rho_{1}\right) \sin \left(m \varphi_{1}+\varphi_{0}\right)=\sum_{p=-\infty}^{p=\infty} Z_{p}\left(k_{2} \rho\right) J_{p-m}\left(k_{2} d\right) \sin \left(p \varphi+\varphi_{0}\right),
$$

where $Z=J$ or $Y$

$$
\text { and } K_{m}\left(k_{4} \rho_{1}\right) \sin \left(m \varphi_{1}+\varphi_{0}\right)=\sum_{p=-\infty}^{p=\infty} K_{p}\left(k_{4} \rho\right) I_{p-m}\left(k_{3} d\right) \sin \left(p \varphi+\varphi_{0}\right)
$$

Using Eq. (5) and Eq. (31), wavefunction in region II about origin O comes as

$$
\begin{aligned}
& \psi_{I I}(\rho, \varphi, z)= \\
& \sum_{m=0}^{\infty} \sum_{p=-\infty}^{p=\infty}\left[B_{m} J_{p}\left(k_{2} \rho\right)+C_{m} Y_{p}\left(k_{2} \rho\right)\right] J_{p-m}\left(k_{2} d\right) \sin \left(p \phi+\phi_{0}\right) e^{i \beta z}
\end{aligned}
$$

And, using Eq. (7) and Eq. (32), the wavefunction IV about origin O comes as

$$
\psi_{I V}(\rho, \varphi, z)=\sum_{m=0}^{\infty} \sum_{p=-\infty}^{p=\infty} F_{m} K_{p}\left(k_{4} \rho\right) I_{p-m}\left(k_{4} d\right) \sin \left(p \phi+\phi_{0}\right) e^{i \beta z}
$$

Substituting the $\psi_{I I}$ and $\psi_{I V}$ given by Eq. (33) and Eq. (34) into boundary conditions given by Eq. (29) and Eq. (30) and expanding $\sin \left(p \phi+\phi_{0}\right)$ as Fourier series in $\phi$ and then using Eq. (14) yields a set infinite simultaneous equations in $\mathrm{C}_{\mathrm{m}}$ 's as

$$
\sum_{n=0}^{\infty} \sum_{m=0}^{\infty} Q_{m n} C_{m}=0
$$


where

$$
\begin{aligned}
& Q_{m n}=\left[H_{m}\left(k_{1} k_{2}\right) S_{n}\left(k_{2}, k_{4}\right)+G_{m}\left(k_{1} k_{2}\right) T_{n}\left(k_{2}, k_{4}\right)\right] W_{m n}\left(k_{2} d\right) \\
& \text { with } S_{n}\left(k_{2}, k_{4}\right)=\left[k_{2} m_{4}{ }^{*} J_{n}^{\prime}\left(k_{2} b\right) K_{n}\left(k_{4} b\right)-k_{4} m_{2}^{*} J_{n}\left(k_{2} b\right) K_{n}^{\prime}\left(k_{4} b\right)\right] \\
& T_{n}\left(k_{2}, k_{4}\right)=\left[k_{2} m_{4}{ }^{*} K_{n}\left(k_{4} b\right) Y_{n}^{\prime}\left(k_{2} b\right)-k_{4} m_{2}{ }^{*} K_{n}^{\prime}\left(k_{4} b\right) Y_{n}\left(k_{2} b\right)\right] \\
& \text { and } W_{m n}\left(k_{2} d\right)=J_{n-m}\left(k_{2} d\right)+(-1)^{m+1} \cos 2 \varphi_{0} J_{n+m}\left(k_{2} d\right)
\end{aligned}
$$

\section{Results and Discussion}

The Eq. (24) and Eq. (35) are infinite simultaneous equation in $\mathrm{C}_{\mathrm{m}}$ 's and can be expanded as

$$
\begin{aligned}
& P_{11} C_{1}+P_{21} C_{2}+P_{31} C_{3}+\ldots \ldots \ldots \ldots \ldots \ldots . .=0 \\
& P_{12} C_{1}+P_{22} C_{2}+P_{32} C_{3}+\ldots \ldots \ldots \ldots \ldots \ldots=0 \\
& P_{13} C_{1}+P_{23} C_{2}+P_{33} C_{3}+\ldots \ldots \ldots \ldots \ldots \ldots=0 \\
& Q_{11} C_{1}+Q_{21} C_{2}+Q_{31} C_{3}+\ldots \ldots \ldots \ldots \ldots \ldots=0 \\
& Q_{12} C_{1}+Q_{22} C_{2}+Q_{32} C_{3}+\ldots \ldots \ldots \ldots \ldots \ldots . .=0 \\
& Q_{13} C_{1}+Q_{23} C_{2}+Q_{33} C_{3}+\ldots \ldots \ldots \ldots \ldots \ldots=0
\end{aligned}
$$

For non-trivial solution of $\mathrm{C}_{\mathrm{m}}$ 's, the determinant of co-efficient in above equations should be equal to zero. Therefore,

$$
\operatorname{det}\left|\begin{array}{c}
\tilde{P} \\
\tilde{Q}
\end{array}\right|=0
$$

Where, $\tilde{P}$ and $\tilde{Q}$ are transpose of corresponding matrices given by the Eq. (25) and Eq. (36) respectively. For a given SWC, the solution of Eq. (38) will be a polynomial equation in energy eigenvalues $E$ which various roots will give energy eigenvalues of the system. By increasing the order of determinates, the roots converges to specific values. The lowest root gives ground state energy. The lowest root is obtained numerically which converges to third decimal places for $10 \times 10$ determinant entries in Eq. (40). Considering region II is made of GaAs and all other regions I, III and IV are made of $\mathrm{Ga}_{0.7} \mathrm{Al}_{0.3} \mathrm{As}$, we have taken $m_{1}{ }^{*}=m_{3}{ }^{*}=m_{4}{ }^{*}=1.4 m_{2}{ }^{*}, m_{2}{ }^{*}=5.73 \times 10^{-32} \mathrm{~kg}$. For fixed radii a $=6 \mathrm{~nm}$ and $\mathrm{b}=20 \mathrm{~nm}$, barrier potential energies $\mathrm{U}_{1}=\mathrm{U}_{3}=\mathrm{U}_{4}=190 \mathrm{meV}$ and setting $\beta=0$, the ground state energy is calculated by solving numerically Eq. (40) with $10 \times 10$ entries for varying eccentricity $\mathrm{d}=4 \mathrm{~nm}$ to $13 \mathrm{~nm}$ (within allowed range for $\mathrm{d}$ according to used addition theorem in methodology). The result is shown graphically in Fig. 4. The decrease in ground state energy with increase in eccentricity can be justified as result of Uncertainty principle. 


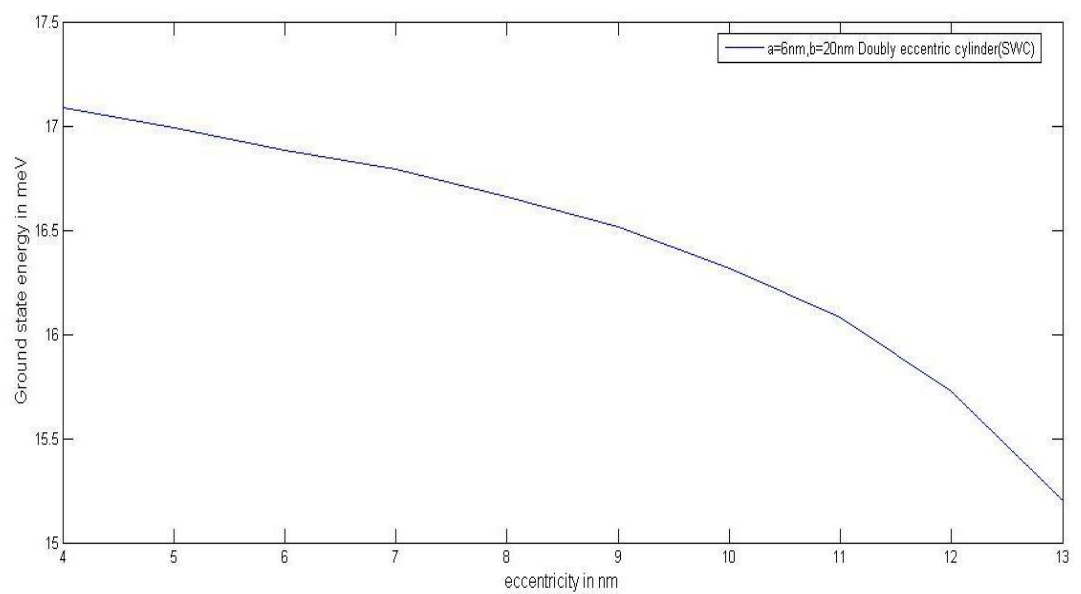

Fig. 4. Variation of ground state energy with eccentricity.

\section{Limiting Behavior}

\subsection{Massive wall confinement (MWC)}

The energy eigenvalues of the system under MWC can be obtained by applying the limit $\frac{m_{i}{ }^{*}}{m_{2}{ }^{*}} \rightarrow \infty, i=1,3$ and 4 during obtaining the solution in section II for SWC. Boundary conditions will now ensure that the quantity $\frac{1}{m_{2}{ }^{*}} \nabla \psi_{I I}=0$ at each boundary between different regions [24]. Repeating the same procedure as in Section II with limit $\frac{m_{i}^{*}}{m_{2}{ }^{*}} \rightarrow \infty, i=1,3$ and 4 , yields two sets of infinite simultaneous equations in $\mathrm{C}_{\mathrm{m}}$, $\mathrm{s}$ as

$$
\begin{aligned}
& \sum_{n=0}^{\infty} \sum_{m=0}^{\infty} P_{m n}^{M W C} C_{m}=0 \\
& \sum_{n=0}^{\infty} \sum_{m=0}^{\infty} Q^{M W C}{ }_{m n} C_{m}=0
\end{aligned}
$$

where

$$
\begin{aligned}
& P_{m n}^{M W C}=\left[\begin{array}{c}
J_{m}^{\prime}\left(k_{2} a\right)\left\{Y_{n+m}\left(k_{2} d_{12}\right)+(-1)^{m-1} \cos \left(2 \phi_{0}\right) Y_{n-m}\left(k_{2} d_{12}\right)\right\} \\
-Y_{m}^{\prime}\left(k_{2} a\right)\left\{J_{n+m}\left(k_{2} d_{12}\right)+(-1)^{m-1} \cos \left(2 \phi_{0}\right) J_{n-m}\left(k_{2} d_{12}\right)\right\}
\end{array}\right] J_{n}^{\prime}\left(k_{2} a\right) \\
& \text { and } Q_{m n}^{M W C}=\left[J_{m}^{\prime}\left(k_{2} a\right) Y_{n}^{\prime}\left(k_{2} b\right)-J_{n}^{\prime}\left(k_{2} b\right) Y_{m}^{\prime}\left(k_{2} a\right)\right] W_{m n}\left(k_{2} d\right)
\end{aligned}
$$




$$
\text { with } W_{m n}\left(k_{2} d\right)=J_{n-m}\left(k_{2} d\right)+(-1)^{m+1} \cos 2 \varphi_{0} J_{n+m}\left(k_{2} d\right)
$$

For non- trivial solution of Eq. (41) and Eq. (42),

$$
\operatorname{det}\left|\begin{array}{c}
\tilde{P}^{M W C} \\
\tilde{Q}^{M W C}
\end{array}\right|=0
$$

Where, $\tilde{P}^{M W C}$ and $\tilde{Q}^{M W C}$ is transpose of the corresponding matrices given by Eq. (43) and Eq. (44) respectively.

\subsection{Hard wall confinement (HWC)}

Under the limit that barrier potential energies (potential energies of region I, III and IV) are infinite, wavefunction $\psi_{I I}$ should vanish at each of the boundary between different regions. Repeating the same procedure discussed in the section II under the boundary condition $\psi_{I I}=0$ at each of the boundaries yields two sets of infinite simultaneous equations in $\mathrm{C}_{\mathrm{m}}$ 's as

$$
\begin{aligned}
& \sum_{n=0}^{\infty} \sum_{m=0}^{\infty} P^{H W C} C_{m}=0 \\
& \sum_{n=0}^{\infty} \sum_{m=0}^{\infty} Q^{H W C}{ }_{m n} C_{m}=0
\end{aligned}
$$

where

$$
\begin{aligned}
& P_{m n}^{H W C}=\left[\begin{array}{l}
J_{m}\left(k_{2} a\right)\left\{Y_{n+m}\left(k_{2} d_{12}\right)+(-1)^{m-1} \cos \left(2 \phi_{0}\right) Y_{n-m}\left(k_{2} d_{12}\right)\right\} \\
-Y_{m}\left(k_{2} a\right)\left\{J_{n+m}\left(k_{2} d_{12}\right)+(-1)^{m-1} \cos \left(2 \phi_{0}\right) J_{n-m}\left(k_{2} d_{12}\right)\right\}
\end{array}\right] J_{n}\left(k_{2} a\right) \\
& \text { and } Q_{m n}^{H W C}=\left[J_{m}\left(k_{2} a\right) Y_{n}\left(k_{2} b\right)-J_{n}\left(k_{2} b\right) Y_{m}\left(k_{2} a\right)\right] W_{m n}\left(k_{2} d\right) \\
& \text { with } W_{m n}(x)=J_{n-m}(x)+(-1)^{m+1} \cos 2 \varphi_{0} J_{n+m}(x)
\end{aligned}
$$

For non- trivial solution of Eq. (47) and Eq. (48),

$$
\operatorname{det}\left|\begin{array}{c}
\tilde{P}^{H W C} \\
\tilde{Q}^{H W C}
\end{array}\right|=0
$$

Where, $\tilde{P}^{H W C}$ and $\tilde{Q}^{H W C}$ is transpose of the corresponding matrices given by Eq. (49) and Eq. (50) respectively.

\section{Conclusion}

A general technique to study electronic states in doubly eccentric cylindrical quantum wire for soft wall confinement has been presented. Massive wall confinement and hard 
wall confinement is presented as its limiting case. The ground state energy of the structure is calculated. The variation in ground state energy with eccentricity is obtained numerically. The ground state energy is found to be decreases with increase in eccentricity of structure. One can similarly find numerically other higher roots of determinant equation to study excited states as well. The discussed method can be also further used to study having more than two similar or different cylindrical barriers nested in it in nano regime.

\section{References}

1. M. Toshipa, Adv. Cond. Matter Phys. 2019, ID 3478506 (2019). https://doi.org/10.1155/2019/3478506

2. P. Kumari, S. Sinha, and L. K. Mishra, J. Pure Appl. Indus. Phys. 7, 264 (2017).

3. A. J. Peter and J. Ebenezar, J. Sci. Res. 1, 200 (2009). https://doi.org/10.3329/jsr.v1i2.1184

4. B. Choupanzadeh, H. Kaatuzian, R. Kohandani, and S. Abdolhosseini, Optics Photonics J. 6, 114 (2016). https://doi.org/10.4236/opi.2016.68B019

5. C. Tablero, J. Appl. Phys. 106, ID 074306 (2009). https://doi.org/10.1063/1.3243290

6. V. I. Boichuk, I. V. Bilynskyi, O. A. Sokolnyk, and I. O. Shakleina, Cond. Matter Phys. 16, ID 33702 (2013). https://doi.org/10.5488/CMP.16.33702

7. C. Tablero, J. Chem. Phys. 122, ID 064701 (2005). https://doi.org/10.1063/1.1844395

8. C. Y. Ngo, S. F. Yoon, W. J. Fan, and S. J. Chua, Phys. Rev. B 74, ID 245331 (2006). https://doi.org/10.1103/PhysRevB.74.245331

9. M. Masale, Physica E: Low-dimensional Systems Nanostruct. 5, 98 (1999). https://doi.org/10.1016/S1386-9477(99)00029-6

10. M. Masale, Physica Scripta 65, 459 (2002). https://doi.org/10.1238/Physica.Regular.065a00459

11. S. Yu and K. W. Kim, J. Appl. Phys. 80, 2815 (1996). https://doi.org/10.1063/1.363199

12. M. Roy and P. A. Maksym, Phys. Rev. B 68, ID 235308 (2003). https://doi.org/10.1103/PhysRevB.68.235308

13. S. D. Sarma and E. H. Hwang, Phys. Rev. B 54, ID 1936 (1996). https://doi.org/10.1103/PhysRevB.54.1936

14. K. A. Matveev, Phys. Rev. B 70, ID 245319 (2004). https://doi.org/10.1103/PhysRevB.70.245319

15. C. Pryor, Phys. Rev. B 44, 12912 (1991). https://doi.org/10.1103/PhysRevB.44.12912

16. S. K. Dey, S. N. Singh, A. Kapoor, and G. S. Singh, Phys. Rev. B 67, ID 113304 (2003). https://doi.org/10.1103/PhysRevB.67.113304

17. G. T. Einevoll, P. C. Hemmer, and J. Thomson, Phys. Rev. B 42, ID 3485 (1990). https://doi.org/10.1103/PhysRevB.42.3485

18. G. T. Einevoll, Phys. Rev. B 42, ID 3497 (1990). https://doi.org/10.1103/PhysRevB.42.3497

19. G. Bastard, Wave Mechanics Applied to Hetrostructures Semiconductor (Les Ulis: Lees Editions de Physique, 1988) pp. 112.

20. G. N. Watson, A Treatise on the Theory of the Bessel Functions (Cambridge University, London, 1958) pp. 361.

21. G. S. Singh and S. N. Singh, J. Math. Phys. 30, 829 (1989). https://doi.org/10.1063/1.528405

22. G. S. Singh and L. S Kothari, J. Math. Phys. 25, 810 (1984). https://doi.org/10.1063/1.528405

23. S. N. Singh and G. S. Singh, J. Math. Phys. 35, 3230 (1994). https://doi.org/10.1063/1.530463

24. P. C. Hemmer and D. T. Wang, Phys. Rev. B 47, ID 6603 (1993). https://doi.org/10.1103/PhysRevB.47.6603 